Importance Weighting 1

IMPORTANCE IS NOT UNIMPORTANT: THE ROLE OF IMPORTANCE WEIGHTING IN QOL MEASURES 


\title{
IMPORTANCE IS NOT UNIMPORTANT: THE ROLE OF IMPORTANCE WEIGHTING IN QOL MEASURES
}

\begin{abstract}
The effect of relative domain importance as a weighting mechanism in quality of life (QoL) measures has been a topic of debate for decades. Studies investigating the role of domain importance in QoL measures have produced mixed results. The mixed results may very well be the consequences of a limited choice of global satisfaction or QoL measures, measurements of domain satisfaction, measurements of domain importance and weighting approaches applied to date. This study investigated the effect of importance weighting without assigning any arbitrary weighting function of domain importance. A cluster analysis was first used to classify individuals into groups, based on their perceived importance of various life domains, and then the relationship between global life satisfaction measure and the composite of domain satisfaction scores between these groups was compared. Results of this study showed that the relationship between global life satisfaction and composite of satisfaction scores did not remain consistent for respondents with different importance rankings of major life domains, which suggested an important effect of domain importance in QoL measures.
\end{abstract}




\section{IMPORTANCE IS NOT UNIMPORTANT: THE ROLE OF IMPORTANCE WEIGHTING IN}

\section{QOL MEASURES}

\section{Introduction}

The role of domain importance in quality of life (QoL) measures as a study focus is by no means a new one (e.g., Hagerty \& Land, 2007; Hagerty et al., 2001; Hsieh, 2003, 2004; Rojas, 2006; Russell \& Hubley, 2005; Russell, Hubley, Palepu, \& Zumbo, 2006). In fact, as Russell and Hubley (2005) indicated, the debate over the role of domain importance was not unique to the area of QoL studies. Studies investigating the role of domain importance have continuously produced mixed results in QoL studies as well as in other areas of studies (e.g., Russell \& Hubley, 2005; Russell et al., 2006; Skalli, Theodossioub \& Vasileioua, 2008).

In the area of life satisfaction studies, researchers have long recognized the possibility that not all aspects of life are equally important to all individuals. "Domain importance" (Campbell, Converse, \& Rogers, 1976), “value priority” (Inglehart, 1978), or “psychological centrality" (Ryff \& Essex, 1992) are all terms used to describe the potential inter-person differences in perceived importance of various life domains. Although there appears to be a consensus that domain importance is not unimportant, debate over the need to incorporate domain importance, otherwise known as importance weighting, into measures of global life satisfaction remains (e.g., Campbell et al., 1976; Hsieh, 2003, 2004; Mastekaasa, 1984; Russell et al., 2006; Wu, 2008a, 2008b; Wu \& Yao, 2006a, 2006b, 2007). Domain importance weighting is generally accomplished in one of the following three ways. One approach is to assign externally determined weights to various domains on a theoretical or conceptual basis. Although these weights reflect the concept of importance, the weights are applied to all respondents without taking into account any potential individual differences. A second approach is to 
consider regression coefficients as weights in a regression analysis when individual domain satisfaction scores are the predictor variables and a global QoL measure is the criterion variable. The results of this approach are heavily dependent on the criterion variable chosen and also do not take into consideration individual respondent differences. A third approach is to apply different domain importance weights to different respondents. This approach of domain importance weighting takes into account the potential individual differences in perceived importance of various domains and is the focus of this study.

Much of the controversy surrounding importance weighting has to do with the fact that many studies found that domain importance as a weighting factor failed to show any detectable increase in the power to explain variations in global QoL measures, in comparison with a simple sum of domain satisfaction scores (e.g., Campbell et al., 1976; Russell et al., 2006; Wu, 2008a, 2008b). For many who chose not to incorporate importance weighting, the decision was not conceptually based but empirically oriented. Opponents of importance weighting include those who purported that importance weighting was conceptually appealing (e.g., Campbell et al., 1976). For example, in their landmark study, Campbell et al. (1976) attempted a number of different scoring systems and techniques of analysis only to find domain importance rating as a weighting factor failed to improve the total variances explained between global QoL instruments and domain satisfaction composites. Based on their empirical findings, Campbell et al. (1976) argued against the use of importance weighting due to the lack of empirical evidence to support a significant effect of domain importance as a weighting factor. As a result, the practice of using a simple sum of satisfaction scores across domains to represent one's global QoL without taking into account the potential inter-person differences has become popular (e.g., Beatty and Tuch, 1997; Mookherjee, 1992). 
Although to a lesser extent, opponents of importance weighting also question the need for incorporating domain importance into global QoL measures from the conceptual basis. The conceptual ground for the opposition of importance weighting can be summarized into two arguments: First, in their call for abandoning the use of multiplicative scores (multiplying satisfaction and importance ratings) to measure QoL, Trauer and Mackinnon (2001) suggested that most QoL measures included domains that were chosen on the basis of consensus, convergence and breadth of coverage. Consequently, domains in most QoL measures should be considered sufficiently universal and should be regarded as already having importance built in to them. Since importance has already been considered in domain selection, importance weighting would not be necessary.

Second, based on an extension of the literature on job satisfaction, specifically Locke's “range-of-affect” hypothesis (Locke, 1969, 1976, 1984), importance weighting has been argued as redundant and unnecessary (e.g., Wu, 2008a, 2008b; Wu \& Yao, 2006a, 2006b, 2007). The range-of-affect hypothesis proposes that satisfaction with specific job facets (or domains) is determined on two subjective factors: 1) the perceived discrepancy between the amount of specific job facets that individuals feel they experience (have) and the amount they want to experience (or the "have-want discrepancy"), and 2) the importance that individuals perceive with various job facets. Based on the range-of-affect hypothesis, satisfaction with a particular job facet is a function of the have-want discrepancy for the job facet and the perceived importance of that job facet (Locke, 1969, 1976). Facet or domain satisfaction is, therefore, influenced by the interaction of the domain have-want discrepancy and domain importance. Especially, at a given level of discrepancy, a domain with high importance can produce a wide range of satisfaction level, while a domain with low importance can only produce a restricted range of satisfaction 
level -- around the center of the satisfaction-dissatisfaction spectrum. Extending the view of the range-of-affect hypothesis, Locke $(1969,1976)$ argued that the relationship between domain satisfaction and overall job satisfaction should be an unweighted additive one. That is, overall job satisfaction should be a sum of satisfactions across various domains of job without the need of weighting domain importance. The position was a natural extension of the range-of-affect hypothesis, as the hypothesis argued that domain satisfaction had already incorporated the judgment of domain importance, hence the concept of implicit weighting, according to Locke (1969, 1976). Weighting domain satisfaction with domain importance would, therefore, be redundant. Extending the range-of-affect hypothesis into the life satisfaction context, opponents of importance weighting (Wu, 2008a, 2008b; Wu \& Yao, 2006a, 2006b, 2007) argued that importance weighting was redundant as importance had already been incorporated into satisfaction evaluation in the life satisfaction context.

However, are the empirical results and conceptual arguments sufficient to warrant the abandonment of importance weighting? Before jumping to any conclusion, a careful examination of the major reasons against importance weighting is necessary. On the empirical ground, the main issue has been that domain importance as a weighting factor often failed to show any significant increase in explaining variations in global QoL measures, compared to the simple sum of domain satisfaction scores. However, the limited performance of importance weighting is likely to be a reflection that most existing evidence against importance weighting has been based on a limited choice of global satisfaction or QoL measures and measurements of domain importance (Russell et al., 2006). In addition, weighting approaches applied so far have mostly centered on the assumption of a linear or a limited curvilinear function of domain importance scores (e.g., Hsieh, 2003, 2004; Rojas, 2006; Wu, 2008a, 2008b; Wu \& Yao, 2006a, 2006b, 
2007). For example, adapting the 12 domains from the World Health Organization Quality of

Life questionnaire (WHOQOL-100), Wu and Yao (2006b) found that in a sample of undergraduate students, importance rating as a weighting mechanism did not improve the correlation between composite of domain satisfaction the Satisfaction with Life Scale (Diner, Emmons, Larsen \& Griffin, 1985). Surveying a group of injection drug users, Russell et al. (2006) evaluated importance weighting using the Injection Drug User Quality of Life Scale (IDUQOL) and found that scores of IDUQOL weighted by importance rating did not perform better than unweighted scores in measuring QoL. Both Wu and Yao's (2006b) and Russell et al.'s (2006) studies assumed a linear function of domain importance rating. On the other hand, surveying a group of older adults, Hsieh $(2003,2004)$ showed that the effect of importance weighting was dependent upon how domain importance was measured (rating vs. ranking), the global QoL measures chosen and the weighting approaches applied. As Hsieh $(2003,2004)$ showed, domain importance ranking as a weighting factor appeared to perform better than domain importance rating and there was evidence to suggest that the function of importance (weighting) might not be a linear one. In fact, whether or not weighted domain satisfaction composites would perform significantly better (than unweighted composites) in predicting (or correlating with) global satisfaction or QoL measures is very likely to be a result of the interplay of a multitude of factors, including, among others, the specific global QoL measure(s) chosen (Russell \& Hubley, 2005; Russell et al., 2006), the way domain importance measured, the domains of life (how exhaustive) included, and the type of weighting approach selected (Hsieh, 2003, 2004). Arguing for the abandonment of importance weighting, using the mixed empirical results regarding the effect of importance weighting, is premature because empirical evidence on importance weighting to date has been based on a limited choice of global satisfaction or QoL 
measures, measurements of domain importance and weighting approaches.

Conceptually, the argument "since importance has already been considered in domain selection process, importance weighting would not be necessary" is somewhat arbitrary. As Hsieh (2004) pointed out, there did not appear to be any conceptual conflict in allowing individuals unique combinations of importance weightings in a pre-determined set of domains to represent global QoL. In other words, just because the domains have been pre-determined to be important, it does not have to mean that they must be equally important to every individual.

The other main argument against importance weighting was based on the range-of-affect hypothesis. However, arguing against importance weighting, citing the range-of-affect hypothesis, is somewhat arbitrary. The main argument of Locke's range-of-affect hypothesis $(1969,1976)$ spoke to the relationships between (the so-called have-want) discrepancy, importance and satisfaction within individual domains (or facets). Regarding the relationships between global satisfaction and domain satisfaction, what was postulated, based on the range-ofaffect hypothesis, was that 1) it would be redundant to weight domain satisfaction with domain importance (the concept of implicit weighting) and 2) global (job) satisfaction should just be the sum of domain satisfactions (Locke, 1969, 1976). The rationale for the additive relationship between domain satisfactions and global satisfaction was less than clear. Specifically, the argument that global satisfaction is the sum of domain satisfactions implicitly assumes that the relationship between satisfaction with various domains is a linear and consistent one. By arguing that global satisfaction is a sum of domain satisfaction across all domains, one assumes that, among others, a consistent relationship that has the nature of perfect substitution between satisfaction across all domains (see Rojas, 2006 for a detailed discussion)

The recent study by Rojas (2006) detailed the limitations and implications of this 
assumed linear relationship (and three other alternative specifications) between global life satisfaction and domain satisfaction. As Rojas (2006) suggested, assuming a linear relationship between global life satisfaction and domain satisfaction would result in a loss of many features of the relationship and would clearly restrict our understanding of life satisfaction. In addition, the concept of implicit weighting, in the life satisfaction context, centers on the relationship between discrepancy, importance and satisfaction within individual life domains, not between or across life domains.

The notion that importance has been included in satisfaction evaluations within a domain does not speak to the relationship of satisfaction between or across domains. In short, the rangeof-affect hypothesis (especially the concept of implicit weighting) focuses primarily on the relationship between global satisfaction, domain satisfaction and domain importance within individual domains. Using this hypothesis as the conceptual ground to argue against domain importance weighting, which focuses primarily on the relationship between global satisfaction, composite of domain satisfactions and domain importance across domains is arbitrary.

In sum, the empirical evidence and conceptual arguments on the role of importance weighting in QoL measures to date have been inconclusive and the role of importance weighting in QoL measures deserves further investigation. The purpose of this study is to examine the effect of importance weighing. As discussed earlier, one of the possible reasons for the mixed results on importance weighting might be that the weighting approaches applied to date have been limited. Given that the potential weighting processes could be complex, this study did not adapt a particular weighting mechanism to approximate the potential function of domain importance. Instead, the effect of importance weighting was examined by a two-step approach: first classifying individuals into groups, based on their perceived importance of various life 
domains, and then comparing the relationship between global life satisfaction measure and the composite of domain satisfaction scores between these groups. The two-step approach used in this study did not make any assumption regarding the potential function of domain importance as a weighting factor. By not making any assumption regarding the function of domain importance, this study not only avoided the issues associated with any arbitrarily hypothesized (usually linear or curvilinear) function of domain importance but also recognized that importance weighting could be a complex process that might not be easily captured by any simple function.

Method

Sample

Data analyzed in this study came from a survey of 112 frail older adults who were the recipients of elderly case management services in a large city in the Midwest region of the United States (see Hsieh [2006] for details). The case management unit provides case management to persons age 60 or older and in need of in-home services. This state-funded program serves about 4,000 clients. Clients of the case management services who could not speak English were excluded from the study. Clients who scored lower than 21 on the MiniMental State Examination (MMSE; Folstein, Folstein, \& McHugh, 1975) were also excluded to avoid any potential problems that were the result of cognitive impairment. A trained graduate research assistant set up interview appointments, and face-to-face structured interviews were conducted by the research assistant at the homes of the participants. Most of the study participants were female (81\%) and African American (92\%). The mean age of the study participants was $76.4(S D=7.3)$, ranging from 62 to 94 . The mean years of schooling completed were $9.8(S D=3.0)$, ranging from 2 to 16 . Most of them were retired $(96 \%)$ and had an annual household income below $\$ 15,000(90 \%)$. 
Measures

Domain satisfaction. Participants were asked to rate their satisfaction in the same manner for each of the following seven domains: health, spare time, financial situation, neighborhood, family life, friendships, and religion. Satisfaction with each domain was measured by responses to a single question. These seven questions were derived from Campbell et al.'s (1976) measures. As suggested by Cummins (1995, 1996), global life satisfaction can be validly and economically measured through seven domains: health, productivity, intimacy, material, safety, community, and emotional. The seven domains included in this study examined all but two of the domains suggested by Cummins $(1995,1996)$, namely safety and productivity. The safety domain has been used predominately to measure life quality of people with an intellectual disability (Cummins, 1996), therefore, it was not included. The productivity, or job, domain was excluded because almost all (over 99 percent) of the study participants did not have jobs.

Domain importance. Domain importance was first measured by the same approach as Campbell et al. (1976). That is, participants were asked to rate the importance of each of the seven domains as: not at all important (1), not too important (2), somewhat important (3), very important (4), or extremely important (5). Unfortunately, as Hsieh (2003, 2004) pointed out, the precision of this approach was limited by its inability to provide direct comparisons when two or more domains receive same ratings. For example, if both health and religion domains are rated as "extremely important," it is not clear whether or not these two domains are actually of equal importance. In this situation, it is unclear if the tie has occurred actually because the respondent perceived equal importance or wither the tie was an artifact of the discrete nature of the measurement. To overcome the issue of imprecision, participants were then asked to compare and rank among the domains with the same rating response options to obtain a rank ordering of 
domains. In other words, this produced a hierarchy of domains from one (most important) to seven (least important) for each respondent. It was possible for domains of equal importance to be so ranked. Domain importance ranking data were used in this analysis.

Global satisfaction. Two measures of global satisfaction were used. One was a singleitem question asking the participants to rate their satisfaction with life as a whole as: completely satisfied (7), somewhat satisfied (6), slightly satisfied (5), neither satisfied nor dissatisfied (4), slightly dissatisfied (3), somewhat dissatisfied (2), or completely dissatisfied (1). In addition to the single-item global satisfaction question, the Satisfaction with Life Scale (SWLS, Diener et al., 1985) was used as a measure of global life satisfaction. SWLS is a five-item global life satisfaction scale. All its items are measured on a seven-point scale, and it has shown good psychometric properties among both young and older adult populations (Diener et al., 1985). The reliability coefficient (Cronbach's $\alpha$ ) was .80 for the current study. Analysis

First, a cluster analysis was used to explore types of perceived importance of various life domains for the study participants. That is, data from the ranking of the seven domains were used to conduct a cluster analysis. The aim of this cluster analysis was to identify a set of groups which both minimize within-group variation and maximize between-group variation, based on perceived importance of various life domains. Initially a hierarchical cluster analysis with the squared Euclidien distance metric and Ward's minimum variance clustering algorithm (Ward, 1963) was conducted. Although Ward's method of clustering has been shown to be one of the better clustering methods in several simulation studies (Milligan \& Cooper, 1987), it also has been shown to be heavily influenced by overall profile elevation (Aldenderfer \& Blashfield, 1984; Milligan \& Cooper, 1987). Under the circumstances, researchers have recommended that a 
two-step cluster analysis be adopted, where a hierarchical method is followed by an iterative partitioning cluster analysis (Aldenderfer \& Blashfield, 1984). The second step of the cluster analysis consisted of an iterative partitioning K-means cluster analysis. Initial seed-points for the K-means cluster analysis were derived from the cluster means on each sub-scale of the shopping orientation generated by the initial Wards method results. The K-means method involves several passes through the data reassigning cases to the cluster centroid to which they are nearest. Kmeans methods have been found to be relatively effective in uncovering the structure of known data sets (Aldenderfer \& Blashfield, 1984). The combination of hierarchical cluster analysis followed by a K-means cluster analysis was meant to minimize the weaknesses of each approach, while maximizing each method's strengths. This two-step approach to cluster analysis is important because it allows for movement of cluster membership from the initial solution derived from the Ward's method analysis. The final cluster membership classifications were then used in the subsequent analyses.

Second, the relationship between global life satisfaction measure and the composite of domain satisfaction scores was compared to examine if there was any significant difference between the clusters obtained through the cluster analysis. Specifically, the correlation between the sum of domain satisfaction and the global life satisfaction measure was calculated for each cluster and then Fisher's Z test (Snedecor \& Cochran, 1989) was used to compare the correlation between clusters. The purpose of the analysis for this second step was to determine whether or not the relationship between global/overall satisfaction and the composite of domain satisfaction scores differed significantly between clusters. If the relationship between global/overall satisfaction and the composite of domain satisfaction scores differed significantly between clusters, then it showed that the relationship between global satisfaction and the composite of 
domain satisfaction scores was dependent upon domain importance. Therefore, the effect of importance weighting should not be rejected.

\section{Results}

Table 1 shows satisfaction and importance ranking of each of the seven major life domains. Based on mean ratings shown on the left-hand side of Table 1, respondents were most satisfied with the religion domain, followed by friendships, family life, neighborhood, spare time, health and financial situation. Based on mean rankings shown on the right-hand side of Table 1, the most important life domain was health, followed, in order, by religion, family life, financial situation, friendships, spare time and neighborhood.

[Table 1 about here]

Using data based on importance ranking, a hierarchical cluster analysis with the squared Euclidien distance metric and Ward's minimum variance clustering algorithm (Ward, 1963) was first conducted and an iterative partitioning K-means cluster analysis was then conducted (Aldenderfer \& Blashfield, 1984). Initial seed-points for the K-means cluster analysis were derived from the cluster means on each of the seven importance rankings generated by the initial Wards method results. The final cluster membership classifications were then used in the subsequent analyses.

Since there is no definitive procedure for determining the optimum number of clusters (Aldenderfer \& Blashfield, 1984), Based on the work of Aldenderfer \& Blashfield (1984) as well as Milligan and Cooper (1985), the hierarchical cluster dendrogram was examined. The hierarchical cluster dendrogram showed that an optimal number of groups was four. The fourcluster solution offered the balance between within group variance and between cluster variance and choosing a solution larger than four clusters would not yield additional distinct groups. This 
heuristic method of subjectively inspecting the dendrogram in determining number of clusters has been widely used in the social sciences (e.g., Aldenderfer \& Blashfield, 1984; Milligan and Cooper, 1985; Zabinski, Norman, Sallis, \& Calfas, 2007). After the initial Ward's method hierarchical clustering was used to identify all possible solutions, a K-means method analysis stipulating four clusters was run. Mean scores for each of the four Ward's method clusters along each item of the shopping orientation measure were then used as the initial cluster centroids for the K-means cluster analysis that followed. The results of the K-means cluster analysis assigned each participant to one of four clusters.

Table 2 depicts the final cluster means and standard deviation for the importance rankings of the seven life domains. Cluster 1 consisted of $19(17 \%)$ respondents. It was labeled "less religious" because of the significant elevation (indicating low importance) on the ranking of religion. Cluster 2 had 17 (15\%) respondents. It was labeled "average" because none of the domain importance rankings deviated significantly from the overall mean. Cluster 3 was the largest cluster, with $46(41 \%)$ respondents. It was labeled "all important" because all the domains were ranked with similar (high) importance without much variation. Cluster 4 contained 30 (27\%) respondents. It was labeled "independent" because the significant elevations (indicating low importance) on the rankings of friendships, family life and neighborhood.

[Table 2 about here]

Table 3 shows the correlations between global life satisfaction and the sum of satisfaction scores across domains by cluster. As shown in Table 3, correlations between the sum of domain satisfaction scores and the single-item global satisfaction ranged from .42 (for cluster 3 ) to .81 (for cluster 1). Correlations between the sum of domain satisfaction scores and Satisfaction with Life Scale (SWLS) ranged from .32 (for cluster 2) to .79 (for cluster 1). Fisher's Z test (Snedecor 
\& Cochran, 1989) was used to compare the correlations to determine if the difference between clusters was statistically significant. According to the results of the Fisher's Z test, the difference between cluster 3 and cluster 1 in the bivariate correlation between the sum of domain satisfaction scores and the single-item global satisfaction was statistically significant $(z=2.32, p$ $<.05)$. In addition, the difference between cluster 3 and cluster 1 in the bivariate correlation between the sum of domain satisfaction scores and the Satisfaction with Life Scale (SWLS) score was statistically significant $(z=2.02, p<.05)$.

[Table 3 about here]

\section{Discussion}

The purpose of this study was to examine the effect of importance weighting in QoL measures. More specifically, this study investigated whether or not the relationship between global life satisfaction and the composite of domain satisfaction varied significantly between respondents with different types of importance ranking of major life domains. Based on a survey of frail older adults, findings of this study showed that the relationship between global life satisfaction and the sum of domain satisfaction scores did not remain the same for all respondents with different types of importance ranking of major life domains. Specifically, after a cluster analysis to classify respondents into four distinct groups based on their importance ranking of seven major life domains, this study found that there was a significant difference between two of the four groups in the correlation between global life satisfaction, either a singleitem global life satisfaction question or the SWLS score, and the sum of domain satisfaction scores.

Before discussing these findings, major limitations of this study should be addressed. First, data of this study came from a survey of frail older adults and the sample size was 
relatively small. Generalizibility of these results may, therefore, be somewhat limited. Second, the job domain was eliminated due to the fact that almost all respondents did not have a job. Although the seven major life domains included in this study covered the majority of the major domains listed in previous research (Cummins, 1995, 1996), the consequences of eliminating a major life domain like the job domain were unclear. Third, cluster analysis used in this study, as an analytical method, has been criticized for the lack of statistical indices to help determine the number of clusters and the sensitivity of clustering algorithm on the results (Bergman \& Magnusson, 1997; Steinley, 2003). However, these findings are still worth noting and have the following three major implications:

First, most empirical evidence on importance weighting to date has been based on limited weighting approaches. However, as many (e.g., Campbell et al., 1976) have recognized, the weighting process may not necessarily be as straightforward as many of the previous studies assumed. That is, the limited weighting approaches applied in previous studies (e.g., Hsieh, 2003, Wu \& Yao, 2006a, 2006b) may not capture the real function by which the role domain importance plays in the relationship between global satisfaction and composite of satisfaction across domains. The topic of "how to weight" should, therefore, be further investigated. Although it is not unreasonable to employ different weighting approaches to explore the potential effect of importance weighting, it is certainly not the only way to assess the effect of importance weighting. Exploring the effect of importance weighting by employing different weighting approaches often requires strict assumptions and conditions. Aside from the assumptions of the weighting functions of domain importance, measurements of domain satisfaction and importance must also be psychometrically adequate for the purpose of constructing the weighting approaches (e.g., Trauer \& Mackinnon, 2001). By not making any 
assumption regarding how domain importance should function, this study recognized that the potential effect of importance weighting might not be a simple one. Using a cluster analysis to divide respondents into smaller groups in which members were similar to each other while different from those in other groups in importance ranking of major life domains, this study took an alternative approach to the investigation of importance weighting other than arbitrarily assigning some kind of weighting function of domain importance. It is important, however, to note that it was not the intent of this study to produce a typology of perceived importance of major life domains, given the small sample size. The intent was to classify the respondents in this study, based on their importance ranking of major life domains.

Second, results of this study indicated that the relationship between global life satisfaction and composite of satisfaction scores did not remain consistent for respondents with different importance rankings of major life domains. These results suggested that the relationship between global life satisfaction and composite of satisfaction scores was not independent of domain importance. In other words, findings of this study did not support the claim that global life satisfaction could be accurately represented by a simple sum of satisfaction scores across life domains without taking into account domain importance. The evidence that the relationship between global life satisfaction and composite of satisfaction scores varied between people with different importance rankings of major life domains provided support for importance weighting in QoL. Findings from this study supplemented Russell et al.'s (2006) investigation in which their findings showed that the mean satisfaction ratings for important domains of Injection Drug User Quality of Life Scale (IDUQL) correlated significantly higher with QoL measures than did the mean satisfaction ratings for unimportant domains. More specifically, Russell et al.'s (2006) study examined the effect of importance weighting based on the comparison of important and 
unimportant domains, which could be regarded as within-person oriented. The current study investigated the effect of importance weighting based on the comparison of individuals with different response types/patterns of domain importance, which could be regarded as betweenperson oriented.

Third, in a recent article, Hagerty and Land (2007) presented an analysis of conditions under which the use of importance weights could improve the accuracy of composite QoL indices. They specified a statistical model for QoL judgments and then provided mathematical proof for two major properties of the model: (a) an optimal composite QoL index was one that used the population or sub-population average importance weights, and (b) in the absence of good information on domain importance, an equal-weighting method could be used and it was a so-called minimax estimator, as it minimized extreme disagreements on a composite QoL index. It seems that property (a) of the model specified by Hagerty and Land (2007) would apply well with the findings from the current study. More specifically, an optimal composite QoL could be formed by using the average importance rankings as weights within the clusters, since clusters were found by minimizing internal variance. In addition, findings of this study are consistent with property (b) of the model specified by Harterty and Land (2007). More specifically, for clusters showing less variability than others (such as cluster 3 as well as cluster 2) in this study, an equal-weighting method (sum of satisfaction scores across domains) showed less variability in the composite QoL scores and thus less correlation with the global life satisfaction scales. It should be noted that although findings from this study provide support for importance weighting, the topic of "how to weight" remains unclear. That is, exactly how importance should be measured and how importance should be incorporated into QoL measures to produce global 
satisfaction scores remain areas that need further investigation on both theoretical/conceptual and empirical grounds.

In sum, the empirical evidence presented in this study points to the importance of considering domain importance in QoL measures. Given that only a limited choice of global satisfaction or QoL measures, measurements of domain satisfaction, domain importance and weighting approaches have been utilized to examine the effect of importance weighting to date, existing evidence on the role of domain importance in QoL measures is far from conclusive and it is too premature to call for abandoning importance weighting in QoL measures without further research on this topic. 


\section{References}

Aldenderfer, M. S., and R. K. Blashfield: 1984, Cluster analysis (Sage, Beverly Hills, California).

Beatty, P. and S. A. Tuch: 1997, 'Race and life Satisfaction in the middle class', Sociological Spectrum 17, pp. 71-90.

Bergman, L. R., and D. Magnusson: 1997, ‘A person-oriented approach in research on developmental psychopathology', Development \& Psychopathology 9, pp. 291-319.

Campbell, A., Converse, P. E., and W. L. Rogers: 1976, The Quality of American Life: Perceptions, Evaluations, and Satisfactions (Russel Sage, New York).

Cummins, R. A.: 1995, 'On the tale of gold standard for life satisfaction', Social Indicators Research 35, pp. 179-200.

Cummins, R. A.: 1996, 'The domains of life satisfaction: An attempt to order chaos', Social Indicators Research 38, pp. 303-328.

Diener, E., Emmons, R.A., Larsen, R.J. and Griffin, S.: 1985, 'The satisfaction with life scale', Journal of Personality Assessment 49, pp. 71-74.

Folstein, M. F., Folstein, S. E., \& McHugh, P. R.: 1975, 'Mini-Mental State: A practical method for grading the cognitive state of patients for the clinician', Journal of Psychiatric Research 12, pp. 189-198.

Hagerty, M. R., Cummins, R. A., Ferris, A. L., Land, K. C., Michalos, A. C., Peterson, M., Sharpe, A., Sirgy, J., \& Vogel, J.: 2001, 'Quality of life indexes for national policy: Review and agenda for research', Social Indicators Research 55, pp. 1-96. 
Hagerty, M. R., \& Land, K. C.: 2007, 'Constructing summary indices of quality of life: A model for the effect of heterogeneous importance weights', Sociological Methods and Research 35, pp. 455-496.

Hsieh, C. M.: 2003, 'Counting importance: The case of life satisfaction and relative domain importance', Social Indicators Research 61, pp. 227-240.

Hsieh, C. M.: 2004, 'To weight or not to weight: The role of domain importance in quality of life measurement', Social Indicators Research 68, pp. 163-174.

Hsieh, C. M.: 2006, 'Using client satisfaction to improve case management services for the elderly', Research on Social Work Practice 16, pp. 605-612.

Inglehart, R.: 1978, 'Value priorities, life satisfaction, and political dissatisfaction among western publics', Comparative Studies in Sociology 1, pp. 173-202.

Locke, E.A.: 1969, 'What is job satisfaction?', Organizational Behavior and Human Performance 4, 309-336.

Locke, E. A.: 1976, 'The nature and causes of job satisfaction', pp. 1297-1349 in Handbook of Industrial and Organizational Psychology, edited by M. D. Dunnette (Rand McNally, Chicago).

Locke, E. A.: 1984, ‘Job satisfaction', pp. 93-117 in Social Psychology and Organizational Behavior, edited by M. Gruneberg and T. Wall (Wiley, London).

Mastekaasa, A.: 1984, 'Multiplicative and additive models of job and life satisfaction', Social Indicators Research 14, pp. 141-163.

Milligan, G. W., and M. C. Cooper: 1985, 'An examination of procedures determining the number of clusters in a data set', Psychometrika 50, pp. 159-179. 
Milligan, G. W., and M. C. Cooper: 1987, 'Methodology review: Clustering methods, Applied Psychological Measurement 11, pp. 329-354.

Mookherjee, H. N.: 1992, 'Perceptions of well-being by metropolitan and nonmetropolitan populations in the United States', Journal of Social Psychology 132, pp. 513-524.

Rojas, M.: 2006, 'Life satisfaction and satisfaction in domains of life: Is it a simple relationship?' Journal of Happiness Studies 7, pp. 467-497.

Russell, L. B. and A. M. Hubley: 2005, 'Importance ratings and weighting: Old concerns and new perspectives', International Journal of Testing 5, pp. 105-130.

Russell, L. B., A. M. Hubley, A. Palepu and B. D. Zumbo: 2006, 'Does weighting capture what's important? Revisiting subjective importance weighting with a quality of life measure', Social Indicators Research 75, pp. 146-167.

Ryff, C. D. and M. J. Essex: 1992, 'The interpretation of life experience and well-being: The sample case of relocation', Psychology and Aging 7, pp. 507-517.

Snedecor, G. W. and Cochran, W. G.: 1989, Statistical Methods (Iowa State University Press, Ames, Iowa).

Skalli, A., I. Theodossioub and E Vasileioua: 2008, 'Jobs as Lancaster goods: Facets of job satisfaction and overall job satisfaction', Journal of Socio-Economics 37, pp. 1906-1920.

Steinley, D.: 2003, 'Local optima in K-means clustering: What you don't know may hurt you', Psychological Methods 8, pp. 294-304.

Trauer, T. and A. Mackinnon: 2001, 'Why are we weighting? The role of importance ratings in quality of life measurement', Quality of Life Research, 10, pp. 579-585.

Ward, J. H.: 1963, 'Hierarchical grouping to optimize and objective function', Journal of the American Statistical Association 58, pp. 236-244. 
Wu, C. H.: 2008a, 'Examining the appropriateness of importance weighting on satisfaction score from range-of-affect hypothesis: Hierarchical linear modeling for within-subject data', Social Indicators Research 86, pp. 101-111.

Wu, C. H.: 2008b, 'Can we weight satisfaction score with importance ranks across life domains?', Social Indicators Research 86, pp. 468-480.

Wu, C. H. and G. Yao: 2006a, 'Do we need to weight item satisfaction by item importance? A perspective from Locke's range-of-affect hypothesis', Social Indicators Research 79, pp. 485-502.

Wu, C. H. and G. Yao: 2006b, 'Do we need to weight satisfaction scores with importance ratings in measuring quality of life?', Social Indicators Research 78, pp. 305-326.

Wu, C. H. and G. Yao: 2007, 'Importance has been considered in satisfaction evaluation: An experimental examination of Locke's range-of-affect hypothesis', Social Indicators Research 81, pp. 521-541.

Zabinski, M. F., Norman, G. J., Sallis, J. F., and K. J. Calfas: 2007, 'Patterns of sedentary behavior among adolescents', Health Psychology 26, pp. 113-120. 
Table 1

Means and Standard Deviations of Satisfaction Rating and Domain Importance Ranking ${ }^{a}$

\begin{tabular}{lcccc}
\hline & \multicolumn{2}{c}{ Satisfaction rating } & \multicolumn{2}{c}{ Importance ranking } \\
& $M$ & $S D$ & $M$ & $S D$ \\
\hline Religion & 6.54 & 0.54 & 1.96 & 1.60 \\
Friendships & 6.25 & 0.61 & 3.07 & 1.94 \\
Family life & 6.03 & 1.04 & 2.06 & 1.44 \\
Neighborhood & 5.92 & 1.22 & 3.56 & 2.28 \\
Spare time & 5.54 & 1.39 & 3.29 & 2.06 \\
Health & & & & \\
Financial situation & 4.24 & 2.04 & 1.42 & 0.83 \\
\hline
\end{tabular}

Note: ${ }^{\mathrm{a}} \underline{\mathrm{n}}=112$

${ }^{\mathrm{b}}$ Lower numbers indicate greater importance. 
Table 2

Cluster Mean and Standard Deviation of Domain Importance Ranking ${ }^{a}$

\begin{tabular}{|c|c|c|c|c|}
\hline & $\begin{array}{c}\text { Cluster } 1 \\
(n=19) \\
M(S D)\end{array}$ & $\begin{array}{c}\text { Cluster } 2 \\
(n=17) \\
M(S D)\end{array}$ & $\begin{array}{c}\text { Cluster } 3 \\
(n=46)\end{array}$ & $\begin{array}{l}\text { Cluster } 4 \\
(n=30)\end{array}$ \\
\hline & & & & \\
\hline Religion & $5.12(1.36)$ & $1.53(1.07)$ & $1.13(0.45)$ & $1.70(0.75)$ \\
\hline Friendships & $4.29(1.36)$ & $3.47(1.07)$ & $1.17(0.44)$ & $5.07(1.26)$ \\
\hline Family life & $3.00(2.12)$ & $1.53(0.80)$ & $1.24(0.71)$ & $3.10(1.16)$ \\
\hline Neighborhood & $5.71(1.10)$ & $3.76(1.15)$ & $1.17(0.44)$ & $5.87(0.82)$ \\
\hline Spare time & $5.18(1.19)$ & $3.53(1.55)$ & $1.28(0.54)$ & $5.07(1.20)$ \\
\hline Health & $1.71(1.05)$ & $1.88(1.36)$ & $1.11(0.38)$ & $1.50(0.63)$ \\
\hline Financial situation & $2.47(1.01)$ & $3.53(1.12)$ & $1.41(0.78)$ & $4.83(1.42)$ \\
\hline
\end{tabular}

Note: ${ }^{\mathrm{a}} \underline{\mathrm{n}}=112$ 
Table 3

Correlations Between Global Life Satisfaction and Composite Domain Satisfaction Measures ${ }^{a}$

\begin{tabular}{lcc}
\hline & Single-item & SWLS \\
\hline $\begin{array}{l}\text { Sum of satisfaction across seven life domains } \\
\text { Cluster 1 }\end{array}$ & .81 & .79 \\
Cluster 2 & .49 & .32 \\
Cluster 3 & .42 & .48 \\
Cluster 4 & .51 & .70 \\
\hline
\end{tabular}

Note: ${ }^{\mathrm{a}} \underline{\mathrm{n}}=112$ 